\title{
Static analysis on spherical magnetically suspending rotor based on finite element
}

\author{
Wang Weijie, Ren Yuan, and Yin Zengyuan \\ Space Engineering University, Beijing 101416, China
}

\begin{abstract}
In this paper, the static mechanical properties of a spherical magnetically suspending rotor are studied based on the finite element analysis method. The structure of the spherical magnetically suspending rotor is designed. The characteristics of the spherical magnetically suspending rotor are analysed and the necessity of static analysis is pointed out. The main content and general flow of statics analysis are discussed. The two working modes (GUI mode and command flow mode based on APDL) of the finite element software ANSYS are compared and analysed. Then the finite element model of the spherical magnetically suspending rotor is established by the command flow method, and the detailed modelling steps are given. On this basis, the static characteristics of the spherical magnetically suspending rotor are simulated and analysed. The simulation results show that the maximum equivalent stress and rigid body displacement of the rotor are within the safety threshold at a design speed of 10000rpm.
\end{abstract}

\section{Introduction}

Compared to flywheels supported by mechanical bearings, magnetically suspending flywheel (MSFW) relies on electromagnetic force to support them. They have the advantages of no friction, low power consumption, high reliability, and long lifespan. They are ideal actuators for spacecraft attitude control ${ }^{[1][2][3]}$. The MSFW is a complex system integrating mechanical, electronic, electromagnetic, and automatic control. Its performance directly affects the overall performance of the attitude control system, and thus the quality, volume and power consumption of the flywheel, output torque magnitude, accuracy, and bandwidth are all strict requirements ${ }^{[4][5]}$. As the core component of the MSFW, the rotor is a key research object. The usual research method is to use finite element software ANSYS to analyze the mechanical properties of the rotor.

The literature ${ }^{[6]}$ discusses the basic method and process of using ANSYS software to analyze the dynamic characteristics of AMB rotor system. The literature [7] performs the finite element modal analysis under the conditions of multiple groups of parameters for a magnetically suspending flexible rotor system, verifying the correctness of the finite element analysis. The literature ${ }^{[8]}$ uses the Subspace method to calculate the first four natural frequencies and mode shapes, and compares them with experimental modal analysis results. The literature ${ }^{[9]}$ uses the Block Lanczos method to extract the first 20 modes of vibration, displacement and natural frequency of the flywheel rotor. The literature ${ }^{[10]}$ uses SolidWorks to establish a three-dimensional model of a magnetic bearing rotor and introduces it into ANSYS workbench for finite element analysis. The magnetically suspending rotors studied in the above documents are all conventional disc or column structures, and the finite element modeling methods are all GUI (Graphical User Interface) or directly imported from third-party software. The literature ${ }^{[11]}$ optimizes the design of the magnetically suspending rotor to reduce its quality.

At present, the research on magnetic bearings ${ }^{[12][13][14]}$ focuses more on how to optimize the configuration relationship between the permanent magnet magnetic circuit and the electromagnetic magnetic circuit to reduce the power consumption and the volume of the magnetic bearing. The rotors that are targeted are usually traditional disc or spoke structure. The literature ${ }^{[15]}$ proposes a spherical MSFW rotor, which realizes full five-degree-of-freedom control of the MSFW rotor and full-channel decoupling among the magnetic bearings. The universal deflection of the rotor can be precisely controlled, but it has not been studied in depth to the static characteristics of a spherical magnetically suspended rotor (SMSR).

In view of the above mentioned research insufficiency, this paper studies the static characteristics of a spherical magnetically suspending rotor based on the finite element analysis method. The structure of the spherical magnetically suspending rotor is designed. The characteristics of the spherical magnetically suspending rotor are analyzed and the necessity of static analysis is pointed out. The main content and general flow of statics analysis are discussed. The two working modes (GUI mode and command flow mode based on APDL) of the

Foundation item: National Natural Science Foundation of China (No. 51605489) 
finite element software ANSYS are compared and analyzed. Then the finite element model of the spherical magnetically suspending rotor is established by the command flow method, and the detailed modeling steps are given. On this basis, the static characteristics of the spherical magnetically suspending rotor are simulated and analyzed. The simulation results show that the maximum equivalent stress and rigid body displacement of the rotor are within the safety threshold at a design speed of $10000 \mathrm{rpm}$.

\section{SMSR Structure Design}

The spherical magnetically suspending rotor is an inner rotor structure. The rotor system is mainly composed of a rotary table, a Lorentz force magnetic bearing rotor, a motor rotor, and an inductance displacement sensor detection disk. The main structure is shown in Figure 1.

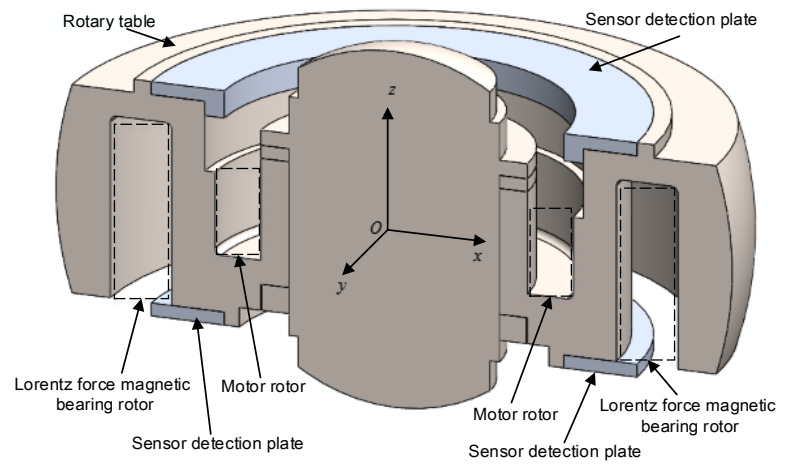

Figure 1. Rotor structure

The motor-driven rotor rotates to output the moment of inertia. The sensor detects the working surface of the disk symmetrically. The inductance displacement sensor is located on the axial outer side of the sensor detection disk and is used for the displacement of the sensitive rotor system along the axial direction. The radial spherical surface is used as the radial displacement of the rotor. The test reference is that four eddy current displacement sensors are arranged on the radial outer side. Compared with the ordinary MSFW rotor, the characteristics of the spherical magnetically suspending rotor are:

(1) From the configuration point of view, the rotor rotary table consists of two spheres with a common sphere centered in the direction of the rotor's polar axis and the radial direction of the equator plane. The two spheres form the envelope of the rotor system. The sphere constitutes the rotor portion of the axial reluctance magnetic bearing and the radial sphere constitutes the rotor portion of the radial reluctance magnetic bearing.

(2) From the material point of view, the entire rotor rotary as a component of the magnetic reluctance magnetic bearing rotor, Lorentz magnetic bearing rotor and motor rotor, the need to provide magnetic medium, so should be selected with high permeability, high saturation Magnetically dense soft magnetic material. At present, commonly used soft magnetic materials mainly include electrical pure iron, iron cobalt vanadium (IJ22) and iron-nickel soft magnetic alloys. Among them, electrical pure iron has advantages such as high saturation magnetic density, low coercive force, and high magnetic permeability, but it is easy to oxidize and rust. The rotor rotary serves as the main body of the rotor. Once rusted, the reliability of the flywheel cannot be guaranteed; In soft magnetic materials, $1 \mathrm{~J} 22$ has the highest saturated magnetic density ${ }^{[16]}$, which is up to $2.1 \mathrm{~T}$. However, the alloy is expensive, easy to oxidize, and has poor processing performance. It is a soft state hardness up to HRB90, and the rotor rotary spherical surface is used as a magnetic bearing. On the other hand, manual grinding is required to achieve the required accuracy. If $1 \mathrm{~J} 22$ is selected, the difficulty and cost of the processing are greatly increased. In the iron-nickel soft magnetic alloy, $1 \mathrm{~J} 50$ has high magnetic permeability and high saturation magnetization (1.56T). Anti-rust performance and processing performance are also good, so select $1 \mathrm{~J} 50$ as the rotary table material.

(3) From a functional point of view, the rotary table as the main body of the rotor, not only to provide most of the rotor moment of inertia, but also as a structural component bearing Lorentz force magnetic bearing rotor, motor rotor and sensor detection disk and other components. Considering that the preliminary design of the motor, Lorentz force magnetic bearing, sensor, axial magnetic bearing, and radial magnetic bearing has been determined, the mechanical performance of the magnetically suspended rotor is more determined by the rotary table. Taking into account the influencing factors of the design speed, moment of inertia, and geometry of the flywheel rotor, the basic structure of the rotary table is finally determined as shown in Figure 2.

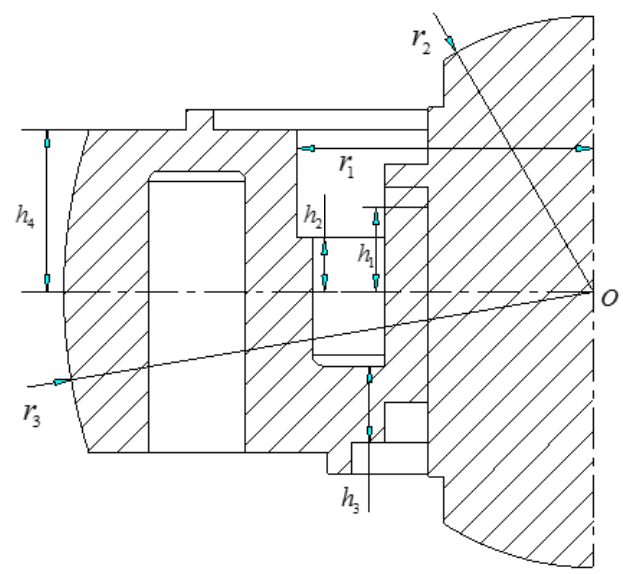

Figure 2. Parameters of rotary table

As the key part of the rotor, the rotary table structural and mechanical properties have the following effects on the flywheel system.

(1) In the high-speed rotation state, the moment of inertia of the rotary table determines the inertia moment of the MSFW. Under the rated speed 10000rpm, the angular momentum of the flywheel output depends on the polar moment of inertia.

(2) Rigid body displacement of the rotary table at high speed directly affects the reliability of the radial magnetic bearing. Spherical MSFW adopts inner rotor structure, especially for radial magnetic bearing, the gyration radius of the rotor is large, and deformation is more likely to occur when rotating at a high speed. Since the air gap 
between the stator and the rotor is only $0.35 \mathrm{~mm}$, the displacement of the rigid body may cause the air gap to change, thereby increasing the difficulty of system control and threatening the safety of the flywheel. This requires the structural design to ensure that the maximum rigid body displacement of the wheel is small enough compared to the air gap within the working speed range of the flywheel. .

(3) As the magnetic medium for each magnetic bearing and motor rotor, the rotary table must ensure sufficient static redundancy for its static performance.

In summary, the core of the spherical magnetically suspending rotor is the rotary table, which is based on the special role of the rotary table in the spherical MSFW rotor system, it is necessary to carry out static analysis.

\section{SMSR Finite Element Modeling}

\subsection{Static model}

The so-called static force is the structure by the static load (fixed load) role, inertia and damping effects can be ignored, under the static load, the structure is in static equilibrium. Static analysis is to calculate the response of a structure under static loading, such as displacement, stress, strain, and reaction force. Static load is an ideal assumption, but in many cases, if the load cycle is much larger than the period of the structure's natural vibration, the inertia effect of the structure can be ignored. This situation can be simplified to static analysis. The static analysis of a SMSR mainly includes displacement (field) analysis, stress (field) analysis. The loads imposed on it mainly include externally applied forces and pressures, steady-state inertial forces, and displacement loads.

Static analysis includes linear analysis and nonlinear analysis ${ }^{[17]}$. Linear static analysis is the most basic but also the most widely used type of analysis for linear elastic materials, static loading conditions. For linear analysis, the material is linear, the stress-strain relationship is linear, the deformation is recoverable, and the small displacement, small strain, and small rotation of the structure occur, and the structural stiffness does not change due to deformation.

According to classical mechanics theory, the general equation of the dynamics of an object is ${ }^{[18]}$

$$
[\boldsymbol{M}]\left\{x^{\prime \prime}\right\}+[\boldsymbol{C}]\left\{x^{\prime}\right\}+[\boldsymbol{K}]\{x\}=\{F(t)\}
$$

Where, $[\boldsymbol{M}]$ is a mass matrix; $[\boldsymbol{C}]$ is a damping matrix; $[\boldsymbol{K}]$ is a stiffness matrix; is a displacement vector; $\left\{x^{\prime}\right\}$ is the velocity vector; $\left\{x^{\prime \prime}\right\}$ is the acceleration vector; $\{F(t)\}$ is the force vector.

In the static analysis, the time-related quantities will be ignored, so the above equation can be simplified as

$$
[\boldsymbol{K}]\{x\}=\{F(t)\}
$$

The general steps of static analysis are usually as follows ${ }^{[19]}$.

(1) Modeling. First, the user should specify the working directory, file name, and analysis title, and then define the model geometry elements, cell types, real constants, material parameters, etc. in the preprocessing.

(2) Set up the solution control. Setting solution control includes defining analysis types, setting general analysis options, specifying load step options, and more. Users can set solution control options through the Solution Control dialog box.

(3) Apply a load.

(4) Solve.

(5) Check the analysis results. The static analysis results are saved in the structural analysis result file and include the following contents: basic solutions (node displacement, temperature, voltage, etc.); derived solutions (node and element stress, node and element strain, element force, node reaction force, etc.).

\subsection{Finite Element Modelling}

ANSYS finite element software provides two working modes: human-computer interaction mode (GUI mode) and command flow input mode ${ }^{[20]}$. The GUI mode does not need to memorize the usage rules of the programming language and the use format of the commands. As long as the mouse picks up the operation on the graphic, it is relatively easy for the beginner to grasp some of them. However, when faced with a complex finite element model, the disadvantages of using the GUI approach will be revealed. For example, there will be a large number of simple and repetitive operations, which will greatly increase the computation time, affect the quality of the model analysis, and generate a large number of data files.

The command flow method (Batch method), ANSYS Parameter Design Language (APDL), is a scripting language that is used to complete finite element routine analysis operations or to establish analysis models through parameterized variables. It uses intelligent analysis. The method provides users with the function of automatically completing the finite element analysis process. That is, the input of the program can be determined according to the specified function, variable, and the selected analysis type. It is the most important basis for completing the optimization design and adaptive grid. APDL allows complex data entry, allowing the user to actually have control of any design or analysis attribute. APDL extends the capabilities beyond the scope of traditional finite element analysis and extends more advanced operations, including sensitivity studies, parametric modelling of parts libraries, design modifications, and design optimization.

Compared with the GUI mode, the command flow is an ASCII text file. Its advantages are: it can reduce a lot of repetitive work, and it is especially suitable for occasions that require repeated calculations after minor modifications; it is not limited by the ANSYS software version and operating system. Easy to save, carry and communicate; users can easily establish a parameterized part library to facilitate the rapid generation of finite element analysis model; use APDL to write some commonly used macro commands to engage in secondary development. In addition, the APDL file system must be used for optimization design and adaptive mesh analysis. 
Based on the above analysis, a finite element model of a magnetically suspending spherical rotor was established using the command flow input method based on APDL. Specific modelling steps are as follows.

Step1, set the unit system, and use the centre of the rotor sphere as the origin of the coordinates, and define the variables as shown in Figure 2.

Step2: The plane model of the rotor rotary table is defined by a combination of points, lines, and planes. The forward profile of the rotary table must be composed of a series of quadrilaterals (edges may be arcs) to ensure the consistency of the topological structure of the wheel, as shown in the Figure. 3, the commands involved include: a point definition command $\mathrm{K}$, a straight line definition command LSTR, an arc definition command LARC, and an area definition command AL.

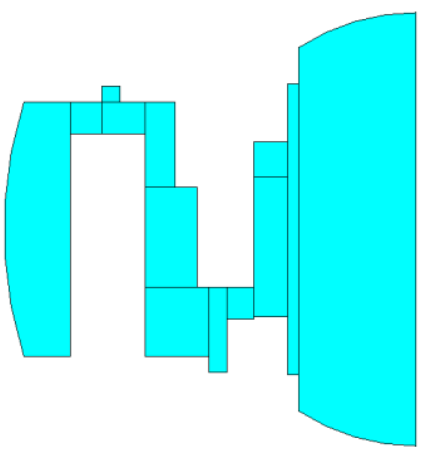

Figure 3. Roulette half-section finite element model

Step3: Use the VROT command to rotate the generator sphere. As shown in Figure 4, it should be noted that the rotor rotary table generated by the rotation is a combination of multiple bodies. Therefore, it is necessary to use the VGLUE command to bond the assembly into one new whole.

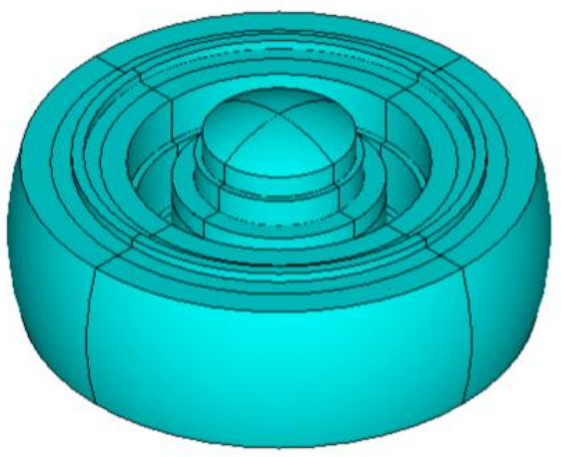

Figure 4. Roulette body finite element model

Step4, set the material properties of 1J50. The unit type is set to Solid185 using the command ET, which is used to construct a three-dimensional, eight-node solid structure, each node having three degrees of freedom in translation along the xyz direction. Use the MP to set the elastic modulus, Poisson's ratio, and density, and associate the material properties with the $3 \mathrm{D}$ model.

Step5, divide the grid. In order to balance the calculation accuracy and time, the grid size is set to $3 \mathrm{~mm}$, and the VSWEEP command is used to divide the grid. The cells formed in this way are almost all regular hexahedron elements, which can effectively guarantee the accuracy of finite element calculations. As shown in Figure 5.

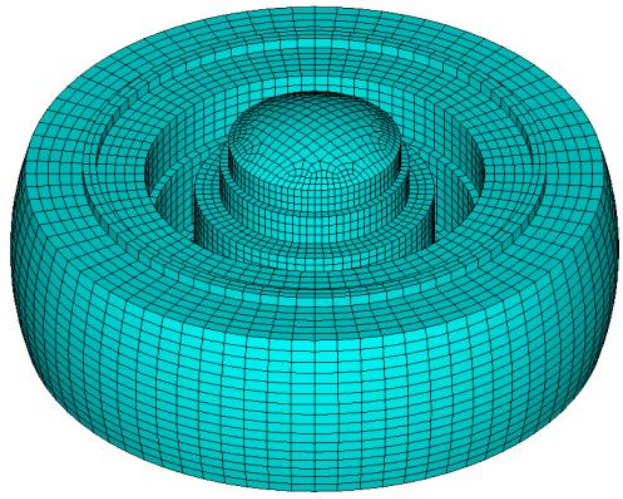

Figure 5. Finite element model of rotary table

Step6, calculate the stress. The inertia release calculation control is set to output only the pre-calculated total mass regardless of inertial release. The type of analysis is set to static analysis. Select the appropriate node, set the constraint type to $3 \mathrm{DOF}$, and use the command OMEGA to set the rotor speed to 10000rpm.

Step7, post-processing. Use the NSORT command to arrange the equivalent stress and displacement vectors in ascending order, and then use the *GET command to extract the maximum equivalent stress and maximum displacement vector, respectively.

\section{Simulation Analysis}

At the rated speed of 10000rpm, the maximum stress distribution of the disk is shown in Figure 6. The maximum equivalent stress obtained from the static analysis is $52.2 \mathrm{MPa}$, which occurs at the radially inner corner of the mounting surface of the upper plate. The yield limit of soft magnetic material $1 \mathrm{~J} 50$ is $147 \mathrm{MPa}$, so the safety factor is 2.8 , which is greater than the design threshold (usually is 2), indicating that the rotary table has sufficient strength and safety redundancy at the design speed.

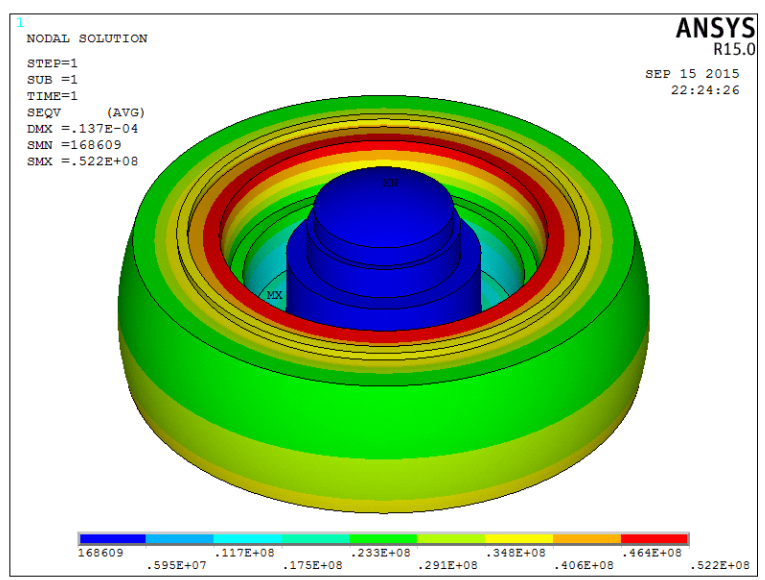

Figure 6. Stress nephogram of rotary table

The rigid disk displacement distribution profile is shown in Figure 7, the maximum rigid body displacement occurs at the lower side of the radial sphere, and the value 
thereof is $0.014 \mathrm{~mm}$. That is, when the rotor is operated at the design speed, the air gap at the lower side of the radial magnetic bearing is reduced by $0.014 \mathrm{~mm}$. The initial air gap of $0.35 \mathrm{~mm}$, air gap reduction rate of $4 \%$, this result is in line with the requirements of engineering practice, indicating that the rotor can maintain the stability of the structure at high speed rotation.

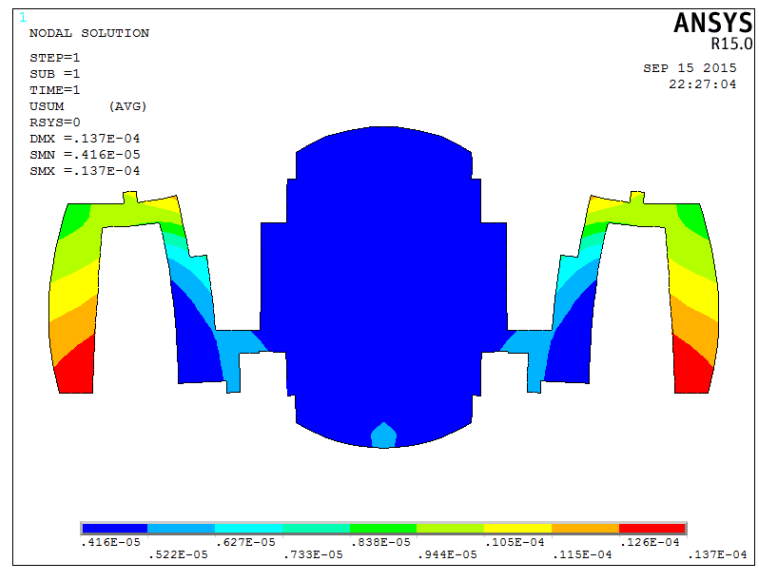

Figure 7. Rigid body displacement of rotary table

\section{Conclusion}

The paper studies the static characteristics of a spherical magnetically suspending rotor based on the finite element analysis method. The design of the spherical magnetically suspending rotor is designed. The characteristics of the spherical magnetically suspending rotor are analyzed and the necessity of static analysis is pointed out. The main content and general flow of statics analysis are discussed. The GUI mode and command flow mode based on APDL of the finite element software ANSYS are compared and analyzed. Then the finite element model of the spherical magnetically suspending rotor is established by the command flow method, and the detailed modeling steps are given. On this basis, the static characteristics of the spherical magnetically suspending rotor are simulated and analyzed. The simulation results show that the maximum equivalent stress and rigid body displacement of the rotor are within the safety threshold at a design speed of 10000rpm.

\section{References}

1. HOU E Y, LIU K, SHAN X Q. Analysis of dynamics characteristics of hybrid magnetically suspending flywheel with oblate external rotor [J]. Journal of Astronautics, 2011, 32(5): 998-1004.

2. WANG X, SHI Y, FAN Y H, et al. A New type of heteropolar radial magnetic bearing biased with permanent magnet for magnetically suspended flywheel [J]. Journal of Astronautics, 2014, 35(12): 1457-1464.

3. WANG $\mathrm{C}$ E, FANG J C, TANG $\mathrm{J}$ Q, et al. Thermal design method and experimental research of magnetically suspended reaction flywheel [J]. Acta Aeronautica et Astronautica Sinica, 2011, 32(4): 598-607.

4. LIU Q, FANG J C, HAN B C. Novel electromagnetic repeated launch locking/unlocking device (RLLUD) based on self-locking for magnetic bearing flywheel [J]. Sensors and Actuators A, 2012(175): 116-126.

5. LIU Q, FANG J C, WU D Y. Optimization and experiment of magnetic bearing wheel rotor $[\mathrm{J}]$. Machinery Design \& Manufacture, 2015(2): 111-114.

6. WAN J G, WANG X P, JIANG P, et al. Application of ANSYS in analyzing dynamic characteristics of active magnetic bearing-rotor System [J]. Machinery Design \& Manufacture, 2009(4): 87-89.

7. WAN J G, WANG X P, GAO Q, et al. Research on Dynamic Characteristics of Rotor System Supported by AMB Based on ANSYS Modal Analysis [J]. Bearing, 2010(6): 1-5.

8. LI K L, XIE Z Y. Model analysis of the magnetically suspending rotor based on ANSYS [J]. Mechanical \& Electrical Engineering Magazine, 2008, 25(1): 1-3.

9. TANG S Q, HUANG P, KE Y W, et al. Modal Analysis of Magnetic bearing Flywheel Rotor Based on ANSYS [J]. Journal of China Three Gorges Univ. (Natural Sciences), 2017, 39(6): 85-89.

10. SONG J C, OUYANG H M, ZHANG G M. Rotor System Of Magnetic Suspending Flywheel Of Dynamic Research Based On Finite Element Analysis [J]. Journal of Mechanical Strength, 2015, 37(3): 403-407.

11. HAN B C, HU G, FANG J C. Optimization design of magnetic suspended gyroscope rotor $[\mathrm{J}]$. Optics and Precision Engineering, 2006, 14(4): 663-666.

12. YOHJI O, HIROAKI K, KOUICHI K. New concept of miracle magnetic bearings[C]. Proceeding of the 9th International Symposium on Magnetic Bearings, USA, August, 3-6, 2004: 89-95.

13. CHRISTIAN E, TILO S, RAINER N. Comparison of active magnetic bearings with and without permanent magnet bias[C]. Proceeding of the 9th International Symposium on Magnetic Bearings, USA, August, 3-6, 2004: 420-425.

14. SUN J J, FANG J C, WANG Xi, et al. A new permanent magnet biased radial magnetic bearing [J]. Transactions of China Electrotechnical Society, 2009, 24(11): 53-60.

15. WANG W J, REN Y, LIU Q, et al. Optimal design of rotary table for spherical rotor of magnetically suspending gimbaling flywheel $[\mathrm{J}]$. Acta Aeronautica et Astronautica Sinica, 2016, 37(9): 2874-2883.

16. China aeronautical materials handbook editorial board. China aeronautical materials handbook (second edition vol.5) [M]. Beijing: China Standard Press, 2002: 176, 187.

17. LIU G Q, YANG Q D. ANSYS Engineering Application Tutorial ANSYS - - Mechanical articles [M].Beijing: China Railway Press, 2003: 185.

18. HUANG Z X, LIU C Z. ANSYS WORKBENCH14.0 Super study manual $[\mathrm{M}]$. Beijing: Post $\&$ Telecom Press, 2013: 167.

19. ZHANG Y Q, ZHANG H C. ANSYS Finite Element Analysis from Getting Started to Proficient [M]. Beijing: Post \& Telecom Press, 2010: 194-195.

20. GONG S G, XIE G L, HUANG Y Q. ANSYS Parametric Programming and Command Manual [M]. Beijing: Machinery Industry Press, 2009: 2-3. 